Aus der Ohren-, Nasen- und Halsklinik der Universität in Gießen. (Direktor: Prof. v. Eicken.)

\title{
Zur Differentialdiagnose zwischen Perichondritis laryngis und Thyreoiditis.
}

\section{Von Prof. Dr. Alfred Brüggemann.}

Vor kurzem hatten wir Gelegenheit, einen Fall von Perichondritis des Kehlkopfes zu beobachten, der wegen seines ungewöhnlichen Verlaufes in diagnostischer Beziehung einige Schwierigkeiten bot.

Ein 26 jähriger Feldwebelleutnant stand im Mai v. J. w'egen Furunkel im rechten äußeren Gehörgàng und anschließender allgemeiner Furunkulose, besonders der Nackengegend, in unserer Klinik in Behandlung. Nach mehreren Wochen heilten die Furunkel ab, sodaB der Mann Anfang Juni geheilt zu seiner Truppe entlassen werden konnte. Am 19. Juni klagte er über Schmerzen beim Schlucken in der Kehlkopfgegend, die Schmerzen nahmen am folgenden Tage so zu, daB schon am Abend kein Tropfen Flüssigkeit mehr geschluckt werden konnte und am nächsten Tage die Aufnahme in unsere Klinik wieder erfolgen muBte. Es sei besonders erwàhnt, daß der Patient früher nie Beschwerden von seiten des Kehlkopfes hatte; auch 14 Tage vorher bei seincr Entlassung aus unserer Klinik wurden Kchlkopf und Lungen gesund befunden.

letzt zeigte sich bei seiner Aufnahme außen am Hals die Gegend des Ring- und Schildkno pels leicht geschwollen und infiltriert. Bcide Knorpel konnten aber noch vor cin ander abgegrenzt werden. Der Ringknorrel war auf Druck empfindlich. Die Thyreoidea zeigte keine Anschwellung oder Druckempfindlichkeit, auch die Haut der vorderen Halsgegend war unverändert.

Laryngoskopisch fand sich eine starke ödematöse Schwellung vornehmlich der rechten ary-epiglottischen Falte und des rechten Ary knornels. Der geschwollene rechte Aryknorpel ragte weit in das Kehlhonflumen vor, sodaß der Einblick in die Tiefe dadurch behindert war. Die linke Seite des Kehlkopfes war anfangs nicht beteiligt, aber schon am nächsten Morgen trat auch eine Schwellung der linken ary eniglottischen Falte und des linken Aryknorpels auf. Auch die orale Fläche der Epiglottis war jetzt, besonders rechterseits, ödematös geschwollen, sodaß sie stark nach links und in den Kehlkopfeingang verdrängt wurde. Es bestand Fieber von 39 ". Die Atemnot war mittlerweile so hochgradig geworden, daß die Tracheotomia inferior gemacht worden mußte. Die Atmung durch die Kanüle ging gut, aber die Schluckbeschwerden bestanden fort. Patient muBte schlieblich durch Klistiere und Kochsalzinfusionen ernährt werden.

Auch einige Tage nach der Tracheotomie hatte die Schwellung im Kehlkopf noch zugenommen. Man sah jetzt kein Kehlkopflumen mehr, sondern nur mehrere ödematöse Wülste, die den Kehlkopfeingang überlagerten. Die Temperatur war auf $38^{\circ}$ zurückgegangen. Als die 
Schwellung im Kehlkopf ihren Höhepunkt erreicht hatte, trat ziemlich plötzlich eine starke Schwellung der ganzen vorderen Halspartie auf. Die anfangs bestehende Infiltration der Gegend des Ring- und Schildknorpels war übergegangen in eine diffuse Schwellung der ganzen Weichteile in der Umgebung der Trachea und des Kehlkopfes, soda $B$ man Einzelheiten nicht mehr fühlen konnte. Die Haut der vorderen Halsseite war blaß, aber deutlich ödematös durchtränkt, sodaß Fingereindrücke etwas bestehen blieben. Die Gegend dcs Ringknorpels war besonders stark druckempfindlich. Unter- und oberhalb des Ringknorpels war die Druckempfindlichkeit wesentlich geringer. Die Thy. reoidea ließ sich nicht abgrenzen, auch sie erschien geschwollen und bei Druck empfindlich. Besonders bei gleichzeitigem Druck gegen die Trachea bzw. den Kehlkopf war die Druckempfindlichkeit ganz aus. gesprochen.

Ein hinzugezogener Chirurg stellte die Diagnose "akute Thyreoiditis“ und empfahl feuchte Umschläge. Nach etwa 3 Tagen ging die Schwellung des Halses zurück, nur blieb noch, wie zu Anfang, eine stärkere Schwellung und Infiltration der Ringknorpelgegend bestehen, schlieBlich aber verschwand auch diese. Gleichzeitig mit der Schwellung am Halse nahm die stark ödematöse Durchtränkung der Schleimhaut im Kehlkopf ab, sodaß die linke Kehlkopfseite bald ganz wieder normal war. Nur die rechte ary-epiglottische Falte und der rechte Aryknorpel blieben noch längere Zeit verdickt, ohne daB es zu einer Abszedierung kam. Endlich war auch diese Schwellung fast ganz zurückgegangen, soda $\beta$ der Patient drei Monate nach Beginn der Erkrankung décanuliert werden konnte. Die Tracheotomiewunde schloß sich sehr schnell. Im Kehlkopf besteht jetzt noch eine leichte Schwellung des rechten Taschenbandes; im übrigen aber ist der Befund völig normal. Der Patient spricht mit leicht heiserer Stimme, ist aber sonst ganz beschwerdefrei.

Während der starken Entzündıngserscheinungen im Kehlkopf fand sich im Urin Eiweiß mit hvalinen Zylindern, auBerdem reichlich Urobilin (Untersuchung der (jieBener Medizinischen Klinik). In diesen Tagen bestand auch vorübergehend eine stärkere Gelbfärbung der Skleren. Eiweiß und Urobilin sind aus dem Urin völlig wieder verschwunden, auch die Skleren sind jetzt normal weib.

Es handelt sich in diesem Fall also um eine hochgradige Perichondritis des Ring- und rechten Aryknorpels, bei der es infolge der Schwere der Infektion sogar zu Leber- und Nierenschädigung vorübergehend kam. Trotzdem heilte die Perichondritis ohne Abszeßbildung aus. Auf der Höhe der Erkrankung trat auBer der Schwellung rechterseits eine starke ödematöse Schwellung der linken Kehlkopfseite (Aryknorpel und ary-epiglottische Falte) auf, sodaB das Kehlkopflumen durch diese Schleimhautschwellungen vorübergehend völlig verlegt wurde. $\mathrm{Ob}$ dem Schleimhautödem der linken Kehlkopfseite auch eine linkseitige Perichondritis zugrundelag, ist nicht wahrscheinlich, es scheint vielmehr diese Schwellung sowie das Oedem der Epiglottis wegen der nur kurzen Dauer als kollaterales entzündliches Oedem von der bestehenden Perichondritis des rechten Aryknorpels auszugehen.

Für die Diagnose der letzteren spricht die lange Zeit bestehende Schwellung dieser Gegend, vor allem auch die dauernde Schleimhautschwellung im subglottischen Raum bei gleichzertiger ausgesprochener Druckempfindlichkeit des Ringknorpels. Die Perichondritis steht höchstwahrscheinlich ätiologisch in Beziehung zu der überstandenen Furunkulose. Eine andere Ursache konnte wenigstens nicht gefunden werden. Die Lungen wurden mehrfach untersucht und stets gesund befunden, auch war der Kehlkopf vorher ohne krankhaften Befund. Es handelt sich demnach wohl um eine septisch-metastatische Perichondritis.

An sich ist das Auftreten einer derartigen Perichondritis nach überstandener Infektion mit Eitererregern, wenn auch selten, so doch schon mehrfach beobachtet. In diesem Fall trat aber ein Symptom auf, das, soweit ich die Literatur übersehe, bisher noch nicht beschrieben wurde Auf der Höhe der Entzündung, als auch die Schwellung im Kehlkopf am stärksten war, stellte sich eine ödematöse Schwellung der ganzen vorderen Halspartie ein, die auch auf die Schilddrüse überging, sodaß der hinzugezogene Chirurg die Diagnose akute Thyreoiditis stellte. Die akute Entzündung der sonst normalen Schilddrüse ist eine verhältnismäßig seltene Erkrankung. Sie ist scharf zu unterscheiden von der akuten Strumitis, der Entzündung einer kropfig entarteten Schilddrüse Letztere ist häufiger und auch wegen der vorhandenen Kropfveränderungen in mancher Beziehung anders verlaufend als die akute Thy reoiditis. In unserem Fall zeigte die Schilddrüse vor der Schwellung keine Veränderungen, sodaß, wenn eine Entzündung der Schilddrüse vorlag, man von Thyreoiditis sprechen muB.

Unsere Kenntnisse uber die akute Thyreoiditis verdanken wir in erster Linie de Quervain. Es gibt eine sogenannte primäre Thy reoiditis, die ohne nachweisbare Erkrankung auftritt, und eine sekundäre, die sich im Verlauf oder im AnschluB einer Erkrankung, meist einer Infektionskrankheit, einstellt. De Quervain unterscheidet weiter zwischen der bei Infektionskrankheiten als Metastase zu deutenden "Thyreoiditis simplex" und der auf die Durchspülungen mit Toxinen zurückzuführenden "toxischen Thyreoiditis“, die auch durch Jod erzeugt werden kann. Daß eine akute Thyreoiditis sekundär nach entzündlichen Prozessen in den oberen Luftwegen auftritt, ist mehr. fach beobachtet worden. So ist sie beschrieben nach Bronchopneumonie und Plcuritis (R. Dunger), nach Angina (Theissen, Stadler, Ewald u. a.), Entzündung an der Zungentonsille (Niedau) und Eite. rungen im Recessus pyriformis (A pelt).

Die Diagnose einer akuten Thyreoiditis stützt sich auf folgende Symptome: Unter Fieberanstieg kommt es zu einer Anschwellung der
Schilddrüse, häufig nur eines Lappens, seltener beider zugleich oder nacheinander. Der entzündete Schilddrüsenlappen ist stark druckempfindlich und läßt sich gegen die Umgebung meistens abgrenzen. Fs bestehen starke Schmerzen beim Schlucken wegen der Bewegung der Schilddrüse beim Schluckakt. Häufig, aber nicht immer, ist dann im weiteren Verlauf der Entzündung Abszeßbildung in der Schilddrüse die Folge. Bei der nicht eitrigen Thyreoiditis, die ja hier nur in Frage kommt, entwickeln sich die angeführten Symptome rasch, klingen dann aber meistens sehr langsam ab, sodaB es oft Wochen dauert, bis die völlige Heilung eingetreten ist. Atembeschwerden sind bei der akuten Thyreoiditis nicht selten. Nach $O$. J. Stein, Menzel $u$. a. is die Dyspnoe bei Thyreoiditis durch Kongestionen der Schleimhaut der oberen Luftwege bedingt. Durch die Untersuchung von Goldmann, der ausgedehnte Anastomosen zwischen den Gefäßen der Schilddrüse und des Kehlkopfes feststellen konnte, ist die sekundäre Beteiligung des Kehlkopfes bei Schilddrüsenerkrankungen und umgekehrt auch die der Schilddrüse bei primären Larynxerkrankungen wohl verständlich.

Es fragt sich nun: liegt in unserem Fall, wie der Chirurg annahm, eine akute Thyreoiditis vor? Darüber besteht kein Zweifel, daß die perichondritisclien Prozesse bei unserem Patienten schon im Kehlkopf vorhanden waren, bevor die starke Schwellung am Halse auftrat, daB diese also sekundär erst hinzukam. Allerdings bestand schon gleich zu Anfang eine geringe entzündliche Infiltration und Schwellung in der Umgebung des Ring- und Schildknorpels, aber die Schilddrüse war anfangs zweifellos ganz unbeteiligt. Im Verlauf einer akuten Perichondritis des Kehlkopfes habe ich in der Literatur keine Thyreoiditis gefunden. Immerhin kann sie natürlich auch hier auftreten. Wegen der ausgedehnıen Anastomosen zwischen den Gefäßen der Schilddrüse und des Kehlkopfes wäre diese Komplikation sogar naheliegend. Sehen wir nun die Symptome der Thyreoiditis in unserem Fall genauer an, so trat bei unserem Patienten plötzlich im Verlauf der Peri. chondritis die starke Schwellung der ganzen vorderen Halspartie ein. Die Thyreoidea war nicht abzugrenzen, überhaupt waren Eunzelheiten am Halse nicht zu fühlen. Dieses Symptom würde nicht gegen eine akute Thyreoiditis sprechen; denn wenn auch gewölınlich die Schilddrüse in ihrem einzelncn Lappen oder als ganze Drüse für sich allein die Schwellung zeigt, so sind doch von einzelnen Autoren auch bei akuter Thyreoiditis diffuse Schwellungen am Halse infolge kollateralen Oedems beschrieben worden (Theissen u. a.). Bei oberflächlicher Untersuchung konnte anch in unserem Falle die Schilddrüse druckreoidea einen Druck ausübte, soda $B$ gleichzeitig die Trachea bzw. der Ringknorpel gedrückt wurde, klagte der Patient über starke Schmerzen. Auch die übrigen angeführten Symptome der Thyreoiditis (Fieber und Schluckbeschwerden) waren vorhanden. Die Atemnot, die bei Thyreoiditis rccht hochgradig sein kann, machte in unserem Fall die Tracheotomie erforderlich. Man könnte daran denken, daß die Thyreoiditis vielleicht durch den Jodanstrich bei der vorausgegangenen Tracheotomie erzeugt wurde. Allerdings spricht dagegen, daB sie erst etwa acht Tage nach dem Jodanstrich auftrat, dieser auch mit Alkohol und Aether nach der Operation wieder entfernt wurde. Auch die plötzlich auftretenden starken Schleimhautschwellungen im Kehlkopf wären als kollatcrales Oedem von der entzündeten Thyreoidea aus wohl zu deuten, wenn sie auch in diesem MaBe bisher noch nicht beobachtet wurden. Jedenfalls scheint zunächst manches für die akute Thyreoiditis zu sprechen.

Trotzdem glauben wir nicht, daB in unserem Fall eine akute Thyreoiditis, wie der Chirurg annahm, bestanden hat. Die Schilddrüse erschien zwar bei oberflachlicher Untersuchung druckempfindlich; wenn man aber daranf achtete, besonders an den seitlichen Schilddrüsenlappen, den Druck nur auf diese und nicht gleichzeitig auf den Kehlkopf auszuüben, so war sie nicht druckempfindlich. Am Isth mus der Thyreoidea erschienen die Partien, die direkt am Ringknorpel lagen, zwar schmerzhaft, aber die weiter nach abwärts zum Jugulum hin gelegenen nicht mehr. Die vorhandene Druckempfindlichkeit war, wie sich bei genauer Untersuchıng herausstellte, nur auf den Ring- und Schildknorpel beschränkt. Dieser Befund spricht für Perichondritıs und gegen Thyreoiditis; denn gerade die Druckempfindlichkeit der ganzen Schilddrise oder eines Lappens ist ein wesentliches Symptom der Thyreoiditis.

Die starke ödematöse Schwellung der vorderen Halspartie würde anch als kollaterales entzündliches Oedem bei Perichondritis genügend erklärt sein, wenn sie auch selten so stark beobachtet wird. In unseremı Falle muf aber wohl eine besonders virulente Infektion vorgelegen haben, wofür die vorübergehende Nierenreizung und Leberschädigang sprichit. Es ist daher ein so starkes kollaterales Oedem, das nicht nur im Kehlkopf, sondern auch aulien zu dieser starken Schwellung fïhrte, hier wohl erklärlich. Auch die kurze Dauer und das so plötzliche Verschwinden dor ödematösen Schwellung der Schilddrüse spricht gegen Thyreoiditis, bei der gerade das Abklingen der Entzündung oft noch Wochen daucrt. Natürlich ist der Uebergang von kollateralem entzündlichen Oedem zur eigentlichen Entzündung eines Organes nur ein kleiner Schritt, und hälffig wird man mikroskopisch schon von Entzündung sprechen, wo man makroskopisch nur Oedem feststellen kann. Wir glauben aber, daß wir nicht berechtigt sind, klinisch schon von akuter Thyreoiditis zu sprechen, so lange sich nur entzindliches Oedem dieser Gegend findet und die Druckempfindlichkeit bei genauer Untersuchung auf dem Kelikopf beschrankt bleibt.

Zusammenfassung. Der Fall lehrt uns, daß unter Umständen im Verlauf einer akıten Perichondritis des Kehlkopfes ödenıatösc Schwel- 
lungszustände außen am Halse auftreten, die das Bild einer akuten Thyreoiditis vortäuschen können. Gegen Thyreoiditis spricht die allein auf den Kehlkopf lokalisierte Druckempfindlichkeit. Die Druckempfindlichkeit der Schilddrüse ist nur scheinbar, da oft gleichzeitig auch auf den Kehlkopf der Druck ausgeübt wird.

Literatur: F. Apelt, M. m. W 1908, Nr. 41. - de Quervain, Mitt. Grenzgeb 2. Supplbd. 1904; 15 1905. - D D nger, M n. W. 1908 S. 1879 Q v. Eisels berg. Deutsche i. Nothnage is Hb. d. spez. Path. u. Ther 22 1896. - Goldmann, Verh. Ver. D. Laryng. 1904. - Kocher, 3 Teil z Pathologie und Therapie des Kropfes D Zschr. f. Chir. 10 1878. K. M.Menze, Arch. f. Laryng $18 \mathrm{~S}$. 53. - N1e da au, XI. KongreB der Italienischen Ges. f. Oto-Laryngo-Rhinologie. Rom, Oktober 1907.-Stadler, M. m. W. 1906 S. $170-0$ J.Ste in, The Laryngoscope. August 1912. Ref. Zhl. f Layyng. 1913 S. $28 .-$ C F. The isen, New York

state Journal of Medecine. Dec. 1913. Ref. Zbl. f Laryng. 1914 S 163. 